\title{
Response deprivation and response satiation as determinants of instrumental performance: Some data and theory
}

\author{
C. DONALD HETH and A. G. WARREN \\ University of Alberta, Edmonton, Alberta, Canada T6G 2E9
}

\begin{abstract}
Sixteen human subjects were presented with an instrumental task in which pressing a button to produce a visual stimulus was followed by an auditory stimulus. Half of the subjects were assigned to a condition under which pressing the button at the subject's operant level produced less of the auditory stimulus than the subject would normally choose to receive. For the others, pressing the button at operant level produced more of the auditory stimulus than the subject would choose. Subjects in the former condition showed increases in instrumental performance; those in the latter showed decreases. The results indicated that the rewarding or punishing effects of an event depend upon the relation of the instrumental contingency to the subject's unconstrained behavior.
\end{abstract}

In one attempt to provide a comprehensive empirical framework for explaining instrumental performance, Premack $(1965,1971)$ has suggested that both the instrumental response and the contingent event be considered in terms of their behavioral characteristics. Using this framework, Timberlake and Allison (1974) have proposed an "adaptive model" of instrumental behavior. The key assumption of this model states that "instrumental performance is a result of a conflict between the freely occurring behavior of the animal and the restrictions of a schedule" (Timberlake \& Allison, 1974 , p. 150). Specifically, the model presupposes that an organism will seek to maintain some optimal level of performance of the contingent behavior. Accordingly, it will modulate other behaviors to maintain this level.

Timberlake and Allison have elaborated this model with respect to one major theorem, which they term the response-deprivation hypothesis. This hypothesis begins by specifying the subject's baseline or operant level of the instrumental and contingent behaviors $\left(\mathrm{O}_{\mathrm{i}}\right.$ and $\mathrm{O}_{\mathrm{c}}$, respectively). The instrumental contingency is then defined in terms of the amount of instrumental responding (symbolized by I) which produces a given amount of the contingent behavior (symbolized by C). Response deprivation is said to exist if

$$
\mathrm{I} / \mathrm{C}>\mathrm{O}_{\mathrm{i}} / \mathrm{O}_{\mathrm{c}}
$$

This investigation was supported by the resources of the Department of Psychology, University of Alberta. We thank Edward Cornell and Douglas Grant for their comments on the manuscript. Requests for reprints should be addressed to $C$. Donald Heth, Department of Psychology, University of Alberta, Edmonton, Alberta, Canada T6G 2E9.
To put this another way, Equation 1 stipulates that, under the contingency, the performance of the instrumental response at its baseline level will allow the subject less than baseline level access to the contingent behavior. The adaptive model of instrumental performance predicts that a subject will increase his instrumental responding under this condition. Several empirical tests have supported this proposal (Allison \& Timberlake, 1974, 1975; Eisenberger, Karpman, \& Trattner, 1967).

It can be seen, however, that response deprivation is only one way in which the subject's performance of the contingent behavior can be disrupted. An interesting alternative is a contingency in which the performance of the instrumental response at its baseline level produces more than the baseline level of the contingent behavior. In the mathematical language used above, the contingency is such that

$$
\mathrm{I} / \mathrm{C}<\mathrm{O}_{\mathrm{i}} / \mathrm{O}_{\mathrm{c}}
$$

This condition, which we will term "response satiation," can be recognized as the converse of the deprivation case." Timberlake and Allison (1974, p. 151) suggest that this condition also produces conflict. Although they did not propose this, it is a simple extension of their model to note that, given a positive contingency, an organism under this condition can resolve the conflict by performing less of the instrumental response.

There is some evidence which supports this hypothesis. Premack (1971) described data collected by Weisman and himself in a situation where forced running was contingent upon a rat's licking of a water tube. In one condition, the subjects spent more time licking than running. Weisman and 
Premack then forced rats to run contingent upon a fixed number of licks. Unfortunately, the contingency was thus defined in units that were different from those of the baseline assessment, which precludes the direct application of Equation 2. However, inspection of Premack's graphs indicates that $\mathrm{I} / \mathrm{C}$ was functionally less than 1.0 when time is used as the unit of measurement. Consistent with the response-satiation hypothesis outlined above, Weisman and Premack observed suppression of licking under this condition. In a more direct test of the adaptive model, Allison and Timberlake (1974) ran rats under a contingency in which licking a $.4 \%$ saccharin solution for a given duration produced an opportunity to lick a $.1 \%$ solution for a specified time. In Experiment 4 of that study, the I/C ratio satisfied Equation 2, and, as the responsesatiation principle would predict, Allison and Timberlake observed suppression of $.4 \%$ licking. Similarly, Allison (1976) and Mazur (1975, 1977) have observed suppression of instrumental behavior under conditions which satisfy Equation 2. However, these latter four studies employed a "reciprocal" contingency, under which access to the instrumental behavior was itself contingent upon completion of the contingent behavior. In the Allison and Timberlake (1974) experiment, for example, the subjects could lick the $.4 \%$ solution only after they had licked $\mathrm{C}$ amount of the $.1 \%$ solution. This arrangement unfortunately departs somewhat from usual instrumental procedures, which limits the generality of these demonstrations.

Since the available empirical evidence is promising, it seems worthwhile to attempt a test of the responsesatiation hypothesis using a more conventional instrumental procedure. Furthermore, the adaptive model makes a somewhat surprising prediction. Equation 1 predicts an outcome similar to that of a reward situation, while Equation 2 predicts one similar to punishment. Thus, a given contingent behavior could potentially serve both as a reward and a punisher of the same instrumental response; the sole determining factor is the relation of $\mathrm{I} / \mathrm{C}$ to $\mathrm{O}_{\mathrm{i}} / \mathrm{O}_{\mathrm{c}}$. Weisman and Premack (Premack, 1971) found evidence which supports this idea. By manipulating water deprivation, they produced baseline licking rates that were lower than forced running. It is again difficult to precisely estimate the $\mathrm{I} / \mathrm{C}$ ratio, but inspection of the data for two subjects indicates the average ratio may have satisfied Equation 1. Although forced running had previously served to punish licking in these subjects, in this condition forced running now served as a reward. Mazur (1975) varied the $\mathrm{I} / \mathrm{C}$ ratio in a reciprocal contingency of drinking and running, and observed similar results. When the I/C ratio was markedly greater than $\mathrm{O}_{\mathrm{i}} / \mathrm{O}_{\mathrm{c}}$, Mazur observed facilitation; when it was less, the same sequence of events produced suppression. The data were not, however, entirely in accord with the prediction of Equation 1 for values of $\mathrm{I} / \mathrm{C}$ which were close to $\mathrm{O}_{\mathrm{i}} / \mathrm{O}_{\mathrm{c}}$.

The focus of the present experiment was to provide an additional test of the predictions given by Equations 1 and 2. Rather than evaluating the relations given by the equations through manipulations of the operant levels of the behaviors as Weisman and Premack did, we varied the $\mathrm{I} / \mathrm{C}$ ratio (cf. Allison, 1976; Mazur, 1975, 1977). The adaptive model outlined above would predict increases in performance when I/C satisfied Equation 1 and decreases when it satisfied Equation 2.

A secondary objective of the investigation was to extend the generality of the response-deprivation hypothesis. Previous statements of this position have emphasized the behavioral characteristics of the events; the Allison and Timberlake (1974, 1975) studies, for example, employed a consummatory response as the contingent behavior. This approach encounters obvious difficulties in situations where the response aspects of the event are minimal or absent (e.g., electrical brain stimulation). Especially problematic is the baseline assessment of a nonbehavioral event. Premack (1971) and Timberlake and Allison (1974) have suggested that this difficulty could be surmounted by using an arbitrary response which produces the event. The present study employed such a strategy.

\section{METHOD}

\section{Subjects}

Sixteen university students (6 females and 10 males), enrolled in introductory psychology courses, participated in this experiment.

\footnotetext{
Apparatus

The experiment was run in a cubicle illuminated by fluorescent lights at $280 \mathrm{~lx}(26 \mathrm{fc})$. The subjects were seated upright at a table. On the table, about $25 \mathrm{~cm}$ in front of the subjects, was a $90 \times 61 \mathrm{~cm}$ panel composed of 64 multicolored lights behind a translucent diffusion screen. In front of this screen was a small box with two pushbuttons marked "audio" and "visual" and a jack for a set of stereo headphones.

Programming equipment, located behind the screen, could deliver either of two stimuli to the subject. The auditory stimulus was a portion of the musical composition "In C" by Terry Riley (Columbia Records: MS 7178) delivered through the headphones at approximately $96 \mathrm{~dB}$ by a continuously running recorder. The composition consists of relatively improvised passages superimposed over a constantly repeating pair of $\mathrm{C}$ notes; it was chosen because it seemed to provide a rather homogeneous auditory stimulus. The visual stimulus consisted of the activation of the colored lights through an electronic device which varied their intensities in synchronization with the recording; this produced a brightly colored and shifting visual pattern. The two stimuli could not be activated at the same time. The experimenter sat by the programming equipment and controlled the timing of the experiment. A television camera
} 
Table 1

Amounts of Visual (V) and Auditory (A) Stimuli (in Seconds) Activated by Deprivation and Satiation Subjects During Baseline and Contingency Periods

\begin{tabular}{|c|c|c|c|c|c|c|c|c|c|c|c|c|}
\hline \multirow[b]{3}{*}{ Subject } & \multicolumn{6}{|c|}{ Deprivation Subjects } & \multicolumn{6}{|c|}{ Satiation Subjects } \\
\hline & \multicolumn{2}{|c|}{ Baseline 1} & \multicolumn{2}{|c|}{ Contingency } & \multicolumn{2}{|c|}{ Baseline 2} & \multicolumn{2}{|c|}{ Baseline 1} & \multicolumn{2}{|c|}{ Contingency } & \multicolumn{2}{|c|}{ Baseline 2} \\
\hline & $\mathrm{V}$ & A & V & A & V & A & V & A & V & A & V & A \\
\hline 1 & 45 & 125 & 160 & 32 & 145 & 40 & 115 & 95 & 45 & 135 & 125 & 80 \\
\hline 2 & 95 & 100 & 170 & 34 & 100 & 100 & 30 & 20 & 10 & 30 & 15 & 20 \\
\hline 3 & 75 & 70 & 150 & 30 & 40 & 130 & 75 & 120 & 40 & 120 & 85 & 105 \\
\hline 4 & 75 & 120 & 155 & 31 & 120 & 80 & 75 & 125 & 35 & 105 & 120 & 50 \\
\hline 5 & 95 & 100 & 165 & 33 & 85 & 115 & 60 & 115 & 40 & 120 & 105 & 105 \\
\hline 6 & 50 & 155 & 150 & 30 & 40 & 140 & 100 & 95 & 45 & 135 & 85 & 85 \\
\hline 7 & 85 & 115 & 160 & 32 & 90 & 100 & 90 & 110 & 40 & 120 & 50 & 140 \\
\hline 8 & 80 & 120 & 165 & 33 & 65 & 145 & 105 & 65 & 40 & 120 & 125 & 50 \\
\hline Mean & 75 & 113 & 159 & 32 & 86 & 106 & 81 & 93 & 37 & 111 & 89 & 79 \\
\hline
\end{tabular}

situated in front of and above the subject allowed the experimenter to monitor the subject's activities.

\section{Procedure}

Subjects were seated and asked to put on the headphones. The purpose of the camera was explained to put them at ease in its presence. After subjects had made themselves comfortable, the first of four separate stages was begun.

Familiarization period. The first stage of the experiment was designed to familiarize the subjects with the operation of the apparatus. During this stage, a push of the "audio" button produced a 5 -sec presentation of the auditory stimulus; a push of the "visual" button produced $5 \mathrm{sec}$ of the visual stimulus. Pushing the button during the stimulus had no effect. This stage lasted $3 \mathrm{~min}$, and was begun by reading the following instructions to the subject: "In this segment of the experiment we are interested in familiarizing you with the apparatus. As you can see it has two buttons. If you want to listen to music you can push the audio button. If you want to see the lights you can push the visual button. Do you understand?"

First baseline period. The next stage was designed to provide an estimate of the subject's operant level of responding for the stimuli. It was identical to the familiarization period, except that its duration was $4 \mathrm{~min}$. At the start, the experimenter read the following instructions: "This section of the experiment is similar to the part you have just completed. In other words, if you wish to hear the music you can push the audio control; if you want to see the lights, you can push the visual control. Do you understand?"'

Contingency period. During this stage of the experiment, the auditory button was disconnected and the auditory stimulus was presented immediately after each activation of the visual stimulus. There were two conditions: For subjects in the deprivation condition, a push of the visual button produced $5 \mathrm{sec}$ of the visual stimulus followed by $1 \mathrm{sec}$ of the auditory stimulus. For those in the satiation condition, pushing the visual button yielded $5 \mathrm{sec}$ of the visual stimulus followed by $15 \mathrm{sec}$ of the auditory. Half of the subjects (three females and five males) were assigned to the deprivation condition; the other half were assigned to the satiation condition. This stage of the experiment was also $4 \mathrm{~min}$ in duration. The instructions for this stage were as follows: "In this segment of the experiment the audio button has been disconnected. A push of the visual control will produce first the lights, and then the music. Do you understand?"

second baseline period. This period was identical to the first baseline period. The subjects were told: "This segment of the experiment is the same as the first two. In other words, if you want to hear the music you may push the audio button, and if you want to see the lights you can push the visual control. Do you understand?"
Following these four stages, the subjects were given a short questionnaire and then briefed concerning the purpose of the experiment.

\section{RESULTS}

The data of interest are the amounts of each stimulus which subjects activated by pressing the buttons. The total exposure durations of both auditory and visual stimuli activated by the subjects over the two baseline and one contingency periods are given in Table 1. As can be seen in Table 1, the subjects activated appreciable amounts of both stimuli during the two baseline periods. The average ratio of visual to auditory exposure for the deprivation subjects was .71 in the first baseline period and 1.10 in the second. Each subject's ratios in both periods were less than 5.0 (the $\mathrm{I} / \mathrm{C}$ ratio of the contingency in this condition), thereby satisfying the criterion for response deprivation. For subjects in the satiation condition, these ratios averaged .99 and 1.30 for the first and second baseline periods, respectively; all of these ratios were greater than .33 and thus satisfied the responsesatiation criterion. The ratios of these two groups did not differ significantly in either baseline period $[\mathrm{U}(8,8)=21.5$ and 22 for the first and second baseline periods, respectively, $\mathrm{ps}>.05$ ].

The principal data concern the instrumental response of activating the visual stimulus. Although there are problems with this approach to be noted below, we will first examine the total amount of visual time activated. As can be seen in Table 1, groups showed similar baseline levels of visual stimulus activation. However, the two groups showed dramatically different reactions to the contingency. All eight subjects exposed to the contingency which imposed response deprivation activated more of the visual stimulus than they did during either operant period. Similarly, every subject exposed to the contingency which imposed response 
satiation showed a decrease in visual activation.

Statistical analyses corroborated this description. While groups did not differ in baseline visual times during the two baseline periods $[\mathrm{U}(8,8)=26$ and 28.5 for the first and second baseline periods, respectively, $\mathrm{ps}>.05$ ], there was no overlap between groups during the contingency period $[\mathrm{U}(8,8)=0$, $\mathrm{p}<.001$, one-tailed]. To evaluate response changes within a group, nonparametric contrasts were run for each group over the three periods (Marascuilo \& McSweeney, 1967). For the deprivation group, response times during the contingency period were greater than the two baseline periods (C.R. $=3.46$, $\mathrm{p}<.01$, one-tailed). ${ }^{2}$ There was no difference in response times across the two baseline periods (C.R. $=0, p>.05)$. Similarly, response times for the satiation subjects were less during the contingency period than during the baseline periods (C.R. = 3.46, p <.01, one-tailed), while baseline periods did not differ (C.R. $=.50, \mathrm{p}>.05$ ).

These data indicate that subjects are sensitive to contingencies which impose response deprivation or response satiation. There are, however, interpretative difficulties in using time as a measure of instrumental performance. One consequence of the instrumental contingency as defined in the present experiment was that subjects received different amounts of the contingent event (the auditory stimulus) during the contingency period relative to the baseline periods. For example, note that subjects in the deprivation condition received substantially less auditory exposure during the contingency period than they did during either baseline period. Since the auditory and visual events were mutually exclusive, deprivation subjects had a greater opportunity to activate the visual display during the contingency period. Conversely, the satiation subjects had less total time available in the contingency period during which they could activate the visual stimulus. Perhaps the results merely reflect these changes in opportunity to make the instrumental response. The problem is particularly acute in the case of the satiation condition, since the maximum amount of visual exposure which a subject could have activated during the 4-min contingency period was $60 \mathrm{sec}$; for most subjects, this value was below their operant level. Although no subject appears to have reached this performance ceiling, part of the response-suppression effect could nevertheless have been due to this limitation.

It should be noted that the general problem is not unique to the present study. It arises whenever the opportunity to perform the instrumental response differs from baseline to contingency periods. For example, previous tests of the response-deprivation hypothesis and related theories have often employed "reciprocal" contingencies in which the opportunity to perform the instrumental behavior is contingent upon completion of the contingent behavior (e.g., Allison, 1976; Mazur, 1975, 1977). Although Allison (1976, p. 190) has considered the possibility that this might impose an absolute ceiling upon the amount of instrumental behavior which a subject could perform, he overlooked a more subtle point. Under a reciprocal contingency, a subject's opportunity to perform the instrumental response will depend upon how quickly he completes the contingent requirement. If this time is appreciable, then the instrumental behavior is measured over a different time base than that of the baseline period.

At issue is how instrumental performance should be evaluated given differential accessibility to the response. Resolution of this question will have to await general agreement concerning the most appropriate measure of instrumental performance. Nevertheless, these considerations suggest the advisability of examining some measure of the instrumental response other than its duration. We therefore chose to examine visual exposure time using a response measure analogous to the subtraction of magazine time from measures of free-operant response rate.

Accordingly, the duration of auditory exposure was subtracted from the 4-min length of each period. The amount of visual exposure was then converted to a proportion of this difference. The mean proportions over the first baseline, contingency, and the second baseline periods were $.60, .77$, and .62 , respectively, for the deprivation group. These were $.58, .31$, and .58 for the satiation group. Thus, proportions demonstrated the same pattern as total time-a result supported by statistical tests. There was no difference between groups in baseline visual times $[\mathrm{U}(8,8)=27.5$ and 37 for the first and second baseline periods, respectively, ps $>.05]$. The groups did differ during the contingency period $[\mathrm{U}(8,8)=0, \mathrm{p}<.001$, one-tailed $]$. Within groups, visual times during the contingency period were higher than the baseline periods for the deprivation subjects and lower for the satiation subjects (C.R.S $=3.08$ and 3.52 , respectively, ps $<.01$, one-tailed). There was no difference in response times across the two baseline periods for either group (C.R.S $=.51$ and .25 for deprivation and satiation groups, respectively, ps $>.05$ ). Thus, facilitation and suppression effects were still obtained when the performance measure was adjusted for differential response availability.

\section{DISCUSSION}

The data of deprivation subjects clearly confirm the prediction of response increases during the contingency period. They replicate previous demon- 
strations by Eisenberger, Karpman, and Trattner (1967) with humans and Allison and Timberlake (1974, 1975) with rats showing increases in instrumental performance under conditions of response deprivation. The present study extends these demonstrations to include events with relatively minor response components; it therefore supports Premack's (1971) contention that sensory events can be usefully integrated into response-based reinforcement principles.

In our view, the most significant data concern the satiation condition. Although Premack (1971) has extended his probability differential hypothesis to punishment, Timberlake and Allison (1974) confined their discussion to reward and escapeavoidance situations. Like Premack's, the present proposal emphasizes a certain symmetry inherent in the underlying principle; it therefore retains the elegant simplicity of the adaptive model while extending it to include punishment-like effects. As can be seen in the data of satiation subjects, this application was quite successful.

The present proposal and Premack's both assert that there is nothing necessarily unique to a punisher. The punishing properties of an event lie, rather, in its interaction with other aspects of the instrumental situation. Weisman and Premack (Premack, 1971) supported this characterization of punishment by demonstrating that one event (forced running) could both reward and punish a response (drinking) when the operant levels of the two were manipulated. The present experiment indicates that such a state of affairs can occur even when the operant levels of the responses remain unchanged. Whether this application can be extended to other aspects of punishment situations is a question for further research.

Equations 1 and 2 were successful in specifying the conditions under which performance would increase or decrease. They do not, however, provide any indication of the terminal or asymptotic level of performance of instrumental contingencies which satisfy them.

It is possible to develop a prediction of asymptotic performance based upon the adaptive model of Timberlake and Allison (1974). According to this model, subjects respond to reduce the disruption in their behavior caused by the instrumental schedule. In the present experiment, we can represent that disruption as the difference between the amount of an event received during the baseline period $O$, and the amount received during the contingency period, $X$. However, while Timberlake and Allison dealt only with disruptions in the contingent event, the instrumental schedule also disrupts other aspects of the situation as well. Although there are several aspects which are disrupted, and several ways of representing the disruption, we will here make the simple assumption that subjects are concerned only with the instrumental and contingent events, and respond to the algebraic sum of the two baselinecontingency discrepancies. Furthermore, since Timberlake and Allison suggest that both positive and negative discrepancies produce conflict, we will assume that subjects attempt to reduce the absolute value of the net discrepancy.

Based upon these considerations, we therefore propose the following model: Let $\mathrm{O}_{\mathrm{i}}$ and $\mathrm{O}_{\mathrm{c}}$ symbolize the amounts of instrumental and contingent events, respectively, which a subject will freely contact during some standard interval. Let $X_{i}$ and $X_{c}$ represent the amount of each event which the subject experiences during a contingency period of the same length. Under a given instrumental contingency, subjects will contact that value of $\mathrm{X}_{\mathrm{i}}$ for which:

$$
\left|\left(\mathrm{O}_{\mathrm{i}}-\mathrm{X}_{\mathrm{i}}\right)+\left(\mathrm{O}_{\mathrm{c}}-\mathrm{X}_{\mathrm{c}}\right)\right|
$$

is minimal. Notice that Expression 3 stipulates that both instrumental and contingent discrepancies determine asymptotic performance. The model therefore recognizes that subjects can approach $O_{c}$ only at the expense of a change in the discrepancy for the instrumental event (see Premack, 1971). Asymptotic performance represents the solution of this conflict.

Expression 3 can be expanded to provide a quantitative expression for asymptotic performance. Consider a situation like the present one in which the contingent event is presented only when it is produced by the instrumental event. Under a contingency in which I amount of the instrumental event produces $\mathrm{C}$ amount of the contingent, $\mathrm{X}_{\mathrm{c}}=$ $(\mathrm{C} / \mathrm{I}) \mathrm{X}_{\mathrm{i}}$. Substituting this into Expression 3, minimizing and solving for $\mathrm{X}_{\mathrm{i}}$, one arrives at:

$$
\mathrm{X}_{\mathrm{i}}=\mathrm{I}\left(\mathrm{O}_{\mathrm{i}}+\mathrm{O}_{\mathrm{c}}\right) /(\mathrm{I}+\mathrm{C}) .
$$

Recently, Allison (1976) proposed a similar principle of asymptotic performance based upon the assumption that subjects conserve some quantity (e.g., energy) apportioned to instrumental and contingent behaviors across baseline and contingency periods. Accordingly, Allison (1976, Equation 10) proposes that

$$
\mathrm{N}(\mathrm{kI}+\mathrm{C})=\mathrm{kO}_{\mathrm{i}}+\mathrm{O}_{\mathrm{c}},
$$

where $\mathbf{N}$ is the number of times the instrumental response is completed and $\mathrm{k}$ is a constant used to equate instrumental and contingent events with respect to the quantity conserved.

Although Equations 4 and 5 have different 
Table 2

Observed and Predicted Amounts of the Visual Stimulus (in Seconds) Activated by Each Subject During the Contingency Period

\begin{tabular}{|c|c|c|c|c|c|c|}
\hline \multirow[b]{3}{*}{ Subject } & \multicolumn{3}{|c|}{ Deprivation Subjects } & \multicolumn{3}{|c|}{ Satiation Subjects } \\
\hline & \multirow[b]{2}{*}{ Observed } & \multicolumn{2}{|c|}{ Predicted } & \multirow[b]{2}{*}{ Observed } & \multicolumn{2}{|c|}{ Predicted } \\
\hline & & $\begin{array}{c}\text { Adaptive/ } \\
\text { Conservation }\end{array}$ & $\begin{array}{l}\text { Reinforcement } \\
\text { Relativity }\end{array}$ & & $\begin{array}{c}\text { Adaptive/ } \\
\text { Conservation }\end{array}$ & $\begin{array}{l}\text { Reinforcement } \\
\text { Relativity }\end{array}$ \\
\hline 1 & 160 & 148 & 120 & 45 & 52 & 45 \\
\hline 2 & 170 & 165 & 139 & 10 & 11 & 6 \\
\hline 3 & 150 & 131 & 88 & 40 & 48 & 41 \\
\hline 4 & 155 & 165 & 139 & 35 & 46 & 37 \\
\hline 5 & 165 & 165 & 137 & 40 & 48 & 41 \\
\hline 6 & 150 & 160 & 113 & 45 & 46 & 37 \\
\hline 7 & 160 & 163 & 134 & 40 & 49 & 43 \\
\hline 8 & 165 & 171 & 140 & 40 & 43 & 31 \\
\hline Mean & 159 & 159 & 126 & 37 & 43 & 35 \\
\hline
\end{tabular}

theoretical foundations, their mathematical expressions are quite similar. There are, however, some differences which can be noted. Our extension of the adaptive model (Expression 3) differs from Allison's model (Equation 5) in that the former expression is minimized. We have developed it in this fashion to allow for situations in which a complete reduction in conflict is not possible. Also, Expression 3, unlike Equation 5, is not specific with respect to sources of $\mathrm{X}_{\mathrm{i}}$ and $\mathrm{X}_{\mathrm{c}}$; it can, therefore, allow for contributions to these qualities other than those of the contingency. This may prove useful in accounting for response-independent presentations of these events (e.g., Rachlin \& Baum, 1972). Finally, Allison's model includes the parameter $\mathrm{k}$ to insure that the events are commensurate. This approach will certainly be required in many situations (cf. Allison, 1976); we prefer at this time, however, to remain neutral with respect to the ultimate form of the model, since other parametric modifications may prove necessary (see below).

In the present experiment, these minor differences are not at issue, and since $N=X_{i} / I$, Equation 4 is identical to Allison's model with $\mathrm{k}=1$. It can be shown that, under Equation 1, the adaptive/conservation model predicts that $\mathrm{X}_{\mathrm{i}}>\mathrm{O}_{\mathrm{i}}$; similarly, under Equation 2, the model predicts that $\mathrm{X}_{\mathrm{i}}<\mathrm{O}_{\mathrm{i}}$. Thus, the model encompasses the responsedeprivation and response-satiation hypotheses as particular instances of the more general expression. It has the further advantage of being able to specify the asymptotic levels of performance for each subject. Accordingly, predictions under Equation 4 were generated for each subject, using the mean values for $\mathrm{O}_{i}$ and $\mathrm{O}_{c}$ from both baseline periods. The predicted levels of instrumental performance under this model are given in the labeled columns of Table 2. Reference to this table will show that these predicted values are quite close to the observed.

The adaptive/conservation model therefore pro- vides a fairly close fit to the obtained values. Nevertheless, one may wish to consider modifications which might improve the fit further or extend its generality. One possible modification would be to allow for differential sensitivity to the two events. Subjects, for example, might be more sensitive to auditory than to visual discrepancies. Another modification might differentially weigh negative and positive discrepancies. Expression 3 could be recast to allow for these possibilities through the use of specific parameters. Although such a strategy does not seem necessary in the present case, it may prove important in extending this model to other situations and species.

Mazur $(1975,1977)$ has developed an alternative to the conservation model by considering the relative rates of reinforcement provided by both instrumental and contingent behaviors. Mazur's model proposes that these behaviors be considered a single class, whose reinforcement value is determined by the values of $I$ and $C$. Applying the reinforcement relativity model (Mazur, 1977, Equation 4) to the present experiment generates the predictions given in the labeled columns of Table 2 . As can be seen in Table 2, this model underestimates the results of the deprivation condition, but is quite close to the data from the satiation condition. Overall, however, the conservation model would seem to provide more accurate predictions of visual performance. Since the principal support for the reinforcement relativity model comes from studies differing form the present one in their use of "reciprocal" or "interdependent" contingencies (Mazur, 1975, 1977), it would be interesting to evaluate the importance of this factor in instrumental schedules.

\section{REFERENCES}

Allison, J. Contrast. induction, facilitation, suppression, and conservation. Journal of the Experimental Analysis of Behavior, 1976, 25, 185-198. 
Allison, J., \& Timberlake, W. Instrumental and contingent saccharine licking in rats: Response deprivation and reinforcement. Learning and Motivation, 1974, 5, 231-247.

Allison, J., \& Timberlake, W. Response deprivation and instrumental performance in the controlled-amount paradigm. Learning and Motivation, 1975, 6, 122-142.

Eisenberger, R., Karpman, M., \& Trattner, J. What is the necessary and sufficient condition for reinforcement in the contingency situation? Journal of Experimental Psychology, 1967, 74, 342-350.

Marascuilo, L. A., \& McSweeney, M. Nonparametric post hoc comparisons for trend. Psychological Bulletin, 1967, 67, 401-412.

MAzur, J. E. The matching law and quantifications related to Premack's principle. Journal of Experimental Psychology: Animal Behavior Processes, 1975, 1, 374-386.

MAZUR, J. E. Quantitative studies of reinforcement relativity. Journal of the Experimental Analysis of Behavior, 1977, 27, 137-149.

Premack, D. Reinforcement theory. In D. Levine (Ed.), Nebraska symposium on motivation: 1965. Lincoln: University of Nebraska Press, 1965.

Premack, D. Catching up with common sense or two sides of a generalization: Reinforcement and punishment. In R. Glaser
(Ed.), The nature of reinforcement. New York: Academic Press, 1971.

RaChIIN, H., \& BaUM, W. M. Effects of alternative reinforcement: Does the source matter? Joumal of the Experimental Analysis of Behavior, 1972, 18, 231-241.

Timberlake, W., \& Allison, J. An empirical approach to instrumental performance. Psychological Review, 1974, 81, 146-164.

\section{NOTES}

1. Alternatively, Equation 2 specifies deprivation of the instrumental behavior. We prefer the term "response satiation" because it emphasizes the consequences of the operation for the contingent response.

2. C.R. $=\hat{\Psi} /[\operatorname{Var}(\dot{\Psi})]^{1 / 2}$

(Received for publication August 30, 1977; revision accepted February 19, 1978.) 